\title{
Testing of Lenses With the Wave Front Reversing Interferometer ${ }^{1}$
}

\section{Kazuo Muraoka ${ }^{2}$}

\author{
Institute for Basic Standards, National Bureau of Standards, Washington, D.C.
}

(January 2, 1966)

\begin{abstract}
The data obtained with a reversing interferometer may be applied to the optical path difference equation for evaluating the aberration constants of a lens. This paper gives the development of optical path difference equations for the reversing interferometer. These equations are applied to results obtained with two lenses and a comparison is made of values from the interferometer, geometrical measurements, and theoretical computation.

The interferometer is also applied to the measurement of chromatic aberration. Some data are included for comparison of the interferometer results with those obtained by other methods.

The advantages of the wave front reversing interferometer over the Twyman interferometer are (a) it can be adjusted to show the effects either of spherical aberration or coma separately and independently of astigmatism even though they all exist together: (b) the size of the reversing interferometer element is independent of the size of the lens to be tested; (c) the adjustments are easier than those of the Twyman type; and (d) the fringes are less vulnerable to vibration effects than most other interferometers.
\end{abstract}

Key Words: Aberration, interferometry, lens testing, mirror testing.

\section{Introduction}

A modification of the Kösters $[1]^{3}$ prism interferometer, labeled "The wavefront reversing interferometer" by J. B. Saunders [2], is used to evaluate the primary monochromatic aberrations of two lenses. The methods and instrumentation for this application were described orally by Saunders [3] but details of the equations and method of use have not been described in the literature.

Equations for optical path difference, for this interferometer, are developed from the corresponding equations derived by Conrady and used by Kingslake [4] with the Twyman interferometer. Results are obtained on a simple aspheric lens and a telescope objective. Because both the application and analysis of results with this interferometer are so simple the amount of data used for these results is very extensive. These results are condensed into tables and graphs to show the good agreement obtainable with this interferometer and results obtained by other methods.

\section{Optical Path Difference Equation}

Consider parallel light falling on the front surface of a lens, such as a telescope objective, and arriving at a point $M$ (fig. 1) displaced from the ideal image point $M_{0}$ in the Petzval surface.

The rectangular coordinate system $(x, y, z)$ has its $z$-axis coincident with the optic axis of the lens and the $x y$-plane coinciding with the back principal plane.

'Based on work performed while the author was a guest worker at the National Bureat of Standards, 1958-1959.

Present address: Geographical Survey Institute, Ministry of Construction, Tokyo, Japan.

Figures in brackets indicate the literature references at the end of this paper.

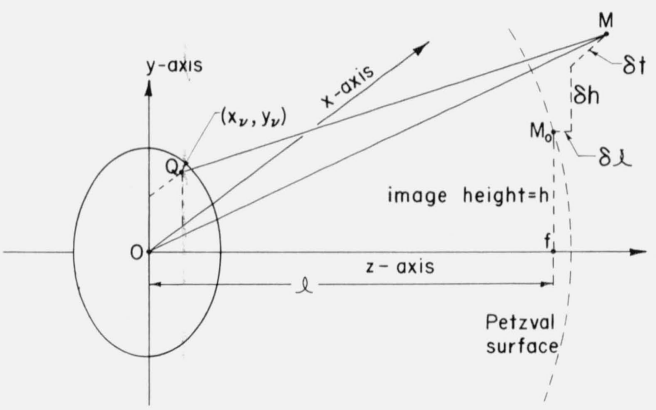

Figure 1. Coordinates for Twyman lens testing interferometer and the Courady optical path difference formula.

The arrows indicate the directions of positive coordinates in the system. The $y z$-plane is chosen as the meridional plane and, therefore, contains the ideal image point $M_{0}$. The coordinates of $M_{0}$ and $M$ are, respectively, $(0, h, l)$ and $(\delta t, h+\delta h, l+\delta l)$.

If we represent the optical path of a ray of light, from the source to $M$, through the point $\left(x_{\nu}, y_{\nu}\right)$ by $F_{\nu}$ and that through the origin by $P_{0}$ the formula [4] for the path difference is,

$$
\begin{gathered}
p_{0}-p_{\nu}=(1 / 4) a_{1}\left(x_{\nu}^{2}+y_{\nu}^{2}\right)^{2}+(h / l) a_{2} y_{\nu}\left(x_{\nu}^{2}+y_{v}^{2}\right) \\
+\left(h^{2} / 2 l^{2}\right) a_{3}\left(x_{\nu}^{2}+3 y_{\nu}^{2}\right) \\
+\left(\delta l / 2 l^{2}\right)\left(x_{\nu}^{2}+y_{\nu}^{2}\right)+(\delta h / l) y_{\nu}+(\delta t / l) x_{\nu} .
\end{gathered}
$$

In this formula $l$ can be replaced by the focal length, $f$, if the lens is a telescope objective. The quantities $a_{1}, a_{2}$, and $a_{3}$ are the absolute constants for a lens 
system of a given type and size, in which higher order aberrations are practically negligible. The constants are connected with the usual geometric measures of aberration by the following equations:

$$
\begin{aligned}
& a_{1}=\frac{\text { Longitudinal spherical aberration }}{s^{2} f^{2}} \\
& a_{2}=\frac{\text { Sagittal coma }}{s^{2} h} \\
& a_{3}=\frac{\text { Distance between the focal lines }}{2 h^{2}}
\end{aligned}
$$

where $2 s$ is the aperture of the lens.

Longitudinal spherical aberration is positive when the paraxial focal length is longer than that of the marginal rays. Coma is positive when the absolute value of the $y$-coordinate of the paraxial image point is smaller than that of the intersection of the rays from the two extremities of the $x$-axis in the lens aperture. In this case the flare extends outward. The distance between the focal lines is positive when the distance from the lens to the tangential image line is shorter than that to the sagittal line.

If the inverting interferometer is adjusted to receive the wave front, in the manner described by Saunders [5] (see fig. 2), the two parts of the wave front (one on each side of the dividing plane) are divided by amplitude division, and a component from one side is superimposed on a component from the other side so that interference is obtained in the regions where the two parts overlap.

This folding of one part of the wave front over onto the other part automatically provides a measure of the optical path difference between two rays that are symmetrical with respect to the beam divider plane. The order of interference observed at any point represents the difference in path between the two rays.

It will be convenient to use a coordinate system, for the reference points in the inverting interferometer, that is displaced and rotated relative to the $x y$-coordinate system shown in figure 1 . The new coordinates will be represented by $X$ and $Y$. See figure 3 . We will choose the line of intersection of the dividing plane of the prism with the $x y$-plane as the $X$-axis.

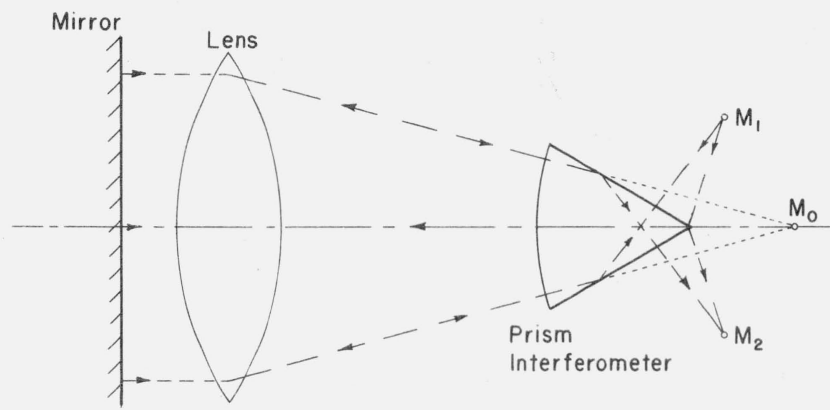

Figure 2. Optics for testing lenses with one conjugate at infinity.

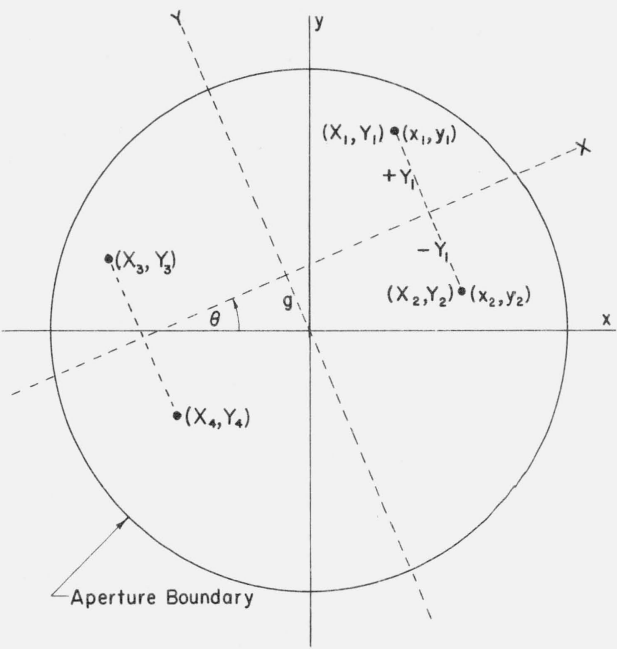

Figure 3. Coordinate system for the $W$ ave Front Reversing Interferometer.

The $Y$-axis will be chosen to pass through the origin of the $x y$-coordinate and normal to the $X$-axis. The angle between the $x$ and $X$-axis is $\theta$. The distance between the origins of the two coordinate systems is $g$.

In general, the $Y$-axis need not be chosen to pass through the origin of the $x y$-axis. It should, however, be normal to the $X$-axis and $g$ is then the distance from the $X$-axis to the origin of the $x y$-coordinates. The above special placement of the $Y$-axis will not restrict the generality of the equations to be derived if the analysis of the data is followed judiciously as described below.

The reversing interferometer prism folds the wave front along the $X$-axis. Any two points in the lens aperture that are symmetrical about the $X$-axis appear to coincide in the interferometer. Let $\left(x_{1}, y_{1}\right)$ and $\left(x_{2}, y_{2}\right)$ be one such pair of points. The corresponding points in the $X Y$-coordinate system are $\left(X_{1}, Y_{1}\right)$ and $\left(X_{2}, Y_{2}\right)$. Because of symmetry about the $X$-axis it is apparent that

and

$$
\left.\begin{array}{l}
X_{1}=X_{2}=X \\
Y_{1}=-Y_{2}=Y
\end{array}\right\} .
$$

Also, it can be shown that the coordinates of one system are related to those of the other system by the following transformation equations:

$$
\left.\begin{array}{l}
x_{1}=X_{1} \cos \theta-Y_{1} \sin \theta-g \sin \theta . \\
y_{1}=X_{1} \sin \theta+Y_{1} \cos \theta+g \cos \theta . \\
x_{2}=X_{2} \cos \theta-Y_{2} \sin \theta-g \sin \theta . \\
y_{2}=X_{2} \sin \theta+Y_{22} \cos \theta+g \cos \theta .
\end{array}\right\}
$$

The path difference for the two rays that appear to pass through $\left(X_{1}, Y_{1}\right)$ is $\left(p_{1}-p_{2}\right)=P_{12}$. Values for 
$p_{1}$ and $p_{2}$ are obtained from eq (1) by putting $\nu$ equal to 1 and 2, respectively. If we then substitute for $x_{1}$, $x_{2}, y_{1}$, and $y_{2}$ the equivalent functions of $X_{1}, X_{2}, Y_{1}$, and $Y_{2}$ shown in eq (3), we obtain an equation for $P_{12}$ as a function of the latter coordinates. On eliminating $X_{2}$ and $Y_{2}$, by means of eqs (2), and dropping subscripts, we obtain

$$
P / 2 Y=A R^{2}+B X+C,
$$

where $R^{2}=X^{2}+Y^{2}$,

$$
\begin{aligned}
& A=a_{1} g+a_{2}(h / f) \cos \theta, \\
& B=2 a_{2} g(h / f) \sin \theta+a_{3}(h / f)^{2} \sin 2 \theta,
\end{aligned}
$$

and $C$ is a function of all quantities in eqs (1), and (3), except the $x, y, X$, and $Y$-coordinates.

All quantities in $A, B$, and $C$ are constant for any one setting (adjustment of the interferometer) and consequently the coefficients themselves are also constant. In general, data from any three reference points are sufficient to evaluate $A, B$, and $C$, but not necessarily $a_{1}, a_{2}$, and $a_{3}$, which are the quantities desired. However, adjustments (values for $g, h$, and $\theta$ ) can be made that will permit the evaluation of the lens constants $a_{1}, a_{2}$, and $a_{3}$. A judicious choice of $g, h$, and $\theta$ will permit the evaluation of any of these constants from only two reference points, that are properly chosen. Data from several reference points will yield statistical values that are more precise than values from only two points.

\section{Analysis of Interferograms}

There are several ways that spherical aberration, coma, or astigmatism may be evaluated separately. Some of the operations for obtaining them are as follows:

\subsection{Spherical Aberration, $a_{1}$}

If the reference points are chosen to fall on the straight line, $X=$ constant, eq (4) becomes:

$$
P / 2 Y=A R^{2}+K_{1},
$$

where $A$ and $K_{1}$ are constants. The slope, $A$, of the straight line $P / 2 Y$ versus $R^{2}$ is readily obtained from two or more reference points. Equation (5) shows $A$ to be a linear function of $g$ and $h$. If $h$ is held constant and values are found for $A$ that correspond to two or more values of $g$, a plot of $A$ versus $g$ will yield a straight line, the slope of which is $a_{1}$.

\subsection{Coma, $a_{2}$}

If $g$ is held constant (eq (5)) and values for $A$ are obtained for two or more values of $h$, a plot of $A$ versus $h$ will yield a straight line whose slope is $\left(a_{2} / f\right) \cos \theta$. Thus the value of $a_{2}$ is readily obtained.

\subsection{Astigmatism, $a_{3}$}

It is apparent that the coefficient of astigmatism, $a_{3}$, appears in eq (6) as a linear function of $B$. The function $B$, may be obtained from observation at any two reference points $\left(X_{1}, Y_{1}\right)$ and $\left(X_{3}, Y_{3}\right)$ such that $Y_{1}=Y_{3}$ (fig. 3) and $X_{1}=-X_{3}$. Equation (4), for these two points becomes:

$$
\begin{aligned}
& A R^{2}+B X_{1}+C=P_{12} / 2 Y_{1}, \\
& A R^{2}-B X_{1}+C=P_{34} / 2 Y_{1},
\end{aligned}
$$

where $P_{34}=\left(P_{3}-P_{4}\right)$. Taking differences, we obtain

$$
B=\left(P_{12}-P_{34}\right) / 4 X_{1} Y_{1}
$$

On dropping the subscripts of $X$ and $Y$, this equation may be written as:

$$
\left(P_{12}-P_{34}\right) / 4 Y=B X .
$$

A plot of $\left(P_{12}-P_{34}\right) / 4 Y$ versus $X$ will approximate a straight line, the slope of which is $B$. If we obtain values of $B$ for different values of $h$, while $g$ is held constant, it is apparent from eq (6) that a plot of the mean value of $B$ (for $h=+h$ and $h=-h$ ) versus $h^{2}$ will yield a straight line, the slope of which is $\left(a_{3} / f^{2}\right)$ $\sin 2 \theta$. Thus the value of $a_{3}$ is directly obtained.

\section{Adjustment of the Dividing Plane}

Because of the analytical form of eq (1) and the manner in which the constants, $a_{1}, a_{2}$, and $a_{3}$ enter it, it is possible to choose the adjustments of the interferometer (values for $g, h$, and $\theta$ ) so as to favor the effect of any of the three aberrations. This is an outstanding advantage of this interferometer. If the source is located on the optic axis of the lens, then $h$, and consequently also $B$, becomes zero (eq 6) and eq (4) becomes:

$$
P / 2 Y=A R^{2}+K_{2},
$$

where $K_{2}$ is invariant with respect to $P, Y$, and $R$. There are no off-axis aberrations and, therefore, the interferogram shows the effect of spherical aberration only.

If $\theta$ is adjusted to equal $\pm 90^{\circ}$ and reference points are confined to the $Y$-axis, eq (4) becomes:

$$
P / 2 Y=a_{1} g R^{2}+K_{3}
$$

where $K_{3}$ is invariant with respect to $P, Y$, and $R$.

If the dividing plane is adjusted to coincide with the $x$-axis, in figure $3(g=0, \theta=0)$, eq (4) becomes:

$$
P / 2 Y=a_{2}(h / f) R^{2}+K_{4}
$$

where $K_{4}$ is invariant with respect to $P, R$, and $Y$. Spherical aberration is eliminated because its effect 
is symmetrical about the optic axis and astigmatism is eliminated because of its symmetry with respect to the $X$-axis. The interferogram contains the effect of coma only.

If the dividing plane is adjusted to bisect the angle between the positive $x$-and $y$-axes $\left(g=0, \theta=45^{\circ}\right)$ eq (4) becomes:

$$
P / 2 Y=a_{2} h / f \sqrt{2} \cdot R^{2}+a_{3}(h / f)^{2} X+K_{5}
$$

where $K_{5}$ is a constant. The term containing $a_{1}$ is eliminated, the term containing $a_{2}$ is reduced to $1 / \sqrt{2}$ of its maximum and the term containing $a_{3}$ is a maximum. The interferogram shows a reduced effect of coma and the full effect of astigmatism.

In these special cases, the analysis can be done easier than for the general setting of the dividing plane. It is, however, preferable to follow the general way of analysis in order to eliminate the error due to locating the origin of the $X Y$-coordinate system (error in $g$ ). The errors in the initial adjustment of the lens (error in $h$ ) is also automatically canceled out by the procedures to be followed. In deriving eq (4), it is assumed that the $Y$-axis passes through the origin of the $x y$-coordinates. Although we are not giving the proof here, it can be shown that this will not affect the generality of the equation as long as the procedures described above are followed.

\section{Experiments}

The prism used in these tests has a $3.8 \mathrm{~cm}$ diam, convex entrance face with a radius of curvature of 20 $\mathrm{cm}$. A light source is placed at the point $M_{1}$ (fig. 2). Light emerges from the curved face of the prism, diverging radially from the virtual source $M_{0}$. The light traverses the lens twice and returns to the point $M_{2}$, which is conjugate to $M_{1}$.

For obtaining different values of $h$, the lens is rotated about an axis parallel to the $x$-axis (fig. 1 ), which coincides with the nodal plane. This position of the axis of rotation avoids shifts in the position of reference points, relative to the lens, when it is rotated. The reference marks (or points) are located between the lens and the mirror.

TABLE 1. Description of lenses

\begin{tabular}{|c|c|c|}
\hline Lens number & I & II \\
\hline Type... & Aspherical-plane & Acromat-doublet \\
\hline Aperture... & $24 \mathrm{~cm}$ & $30 \mathrm{~cm}$ \\
\hline Focal length.... & $200 \mathrm{~cm}$ & $300 \mathrm{~cm}$ \\
\hline Glass.... & BSC -517 & \\
\hline
\end{tabular}

Two lenses are used for these tests. For reference purpose they are described in table 1 .

In these experiments the lenses were adjusted to satisfy the conditions of eqs (9), (10), and (11). However, the analysis is based on the general procedure described above. Values for $A$ and $B$ are obtained by supplying data into eq (4) and then evaluating $a_{1}$, $a_{2}$, and $a_{3}$ from eqs (5) and (6).

In the following tables the distance, $h$, is represented by $\beta$, where $h=f \tan \beta$.

The reference marks used for reading the orders of interference are usually located so that their projections lie on the $Y$-axis, at intervals of $1 \mathrm{~cm}$. However, when taking data for astigmatism it is sometimes convenient to choose reference points elsewhere in the $X Y$-plane.

Since the light traverses the lens twice, its effect on the optical path is doubled. Consequently, the path difference, $P_{12}=\left(p_{1}-p_{2}\right)$ is related to the order of interference, $N$, by the equation

$$
N \lambda=2 P_{12}
$$

where $\lambda$ is the wavelength of the light. The yellow line of helium $(\lambda=0.5876 \mu)$ was used here. The unit used for optical path difference will be $\lambda / 2$, so that $P_{12}$ is numerically equal to the order of interference.

The Kösters prism has a plane wedge [1] built into it. The vertex of this wedge is at a right angle to the dividing plane. In general the line of reference points is chosen either parallel or perpendicular to this wedge. When it is chosen parallel to it (i.e., parallel to the $Y$ axis) a constant may be added to all readings to eliminate the effect of this wedge on the data. If the line of reference points is parallel to the $X$-axis, the wedge must be eliminated by subtracting values from the reading that reduces the effect of the wedge to zero. These values are proportional to the magnitude of the wedge and to the distance from the reference point to the vertex of the wedge. This effect, however, may be regarded as a kind of error in locating the origin of the $X Y$-coordinates with respect to the origin of the $x y$-coordinates (or the optical center of the lens). This error is eliminated by the general procedure of analysis described above.

Figures 4, 5, and 6 show photographs of fringes taken through lens I. Figure 7 shows fringes taken through lens II. The background of fine, low contrast fringes in figure 7 , is caused by light reflected from the two inner surfaces of the air spaced doublet. This light cannot be eliminated except by antireflecting coatings on the surfaces.

To illustrate computation procedures, table 2 shows the observed orders of interference, $N$, at points along the $Y$-axis $(X=0)$ and corresponding positions (values for $Y$ ) of the reference points, observed in figure $6 \mathrm{a}$, column 3 contains values for $P$ (corrected for the prism wedge), in units of $\lambda / 2$.

Figure 8 shows values for $P / 2 Y$ (from column 4 , table 2) plotted against $Y^{2}$ in eq (4). The slope of the best fitting, straight line through these points is the 


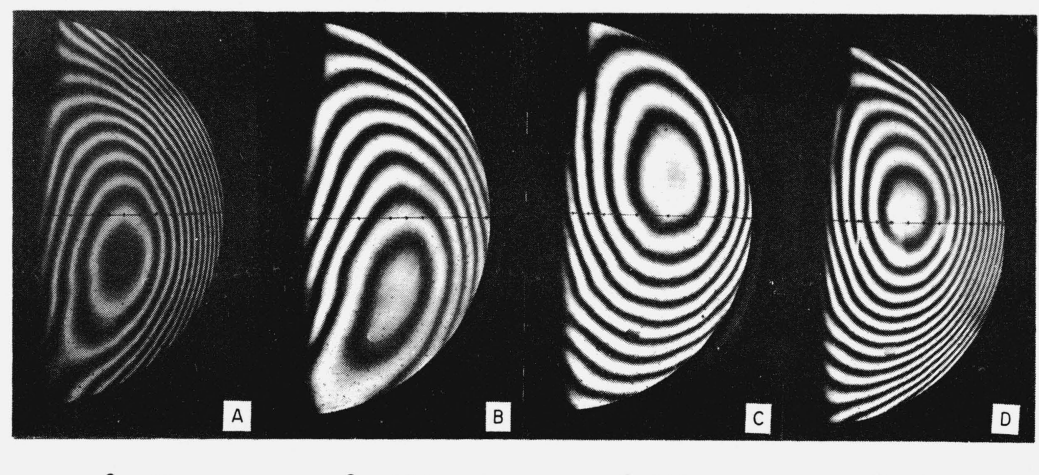

$\beta=-3^{\circ}$

$\beta=-1.5^{\circ}$

$\beta=1.5^{\circ}$

$\beta=3^{\circ}$

Figure 4. Interferograms with Lens No. I, adjusted to $\mathrm{g}=0, \theta=0$, and to different values for $\beta$.

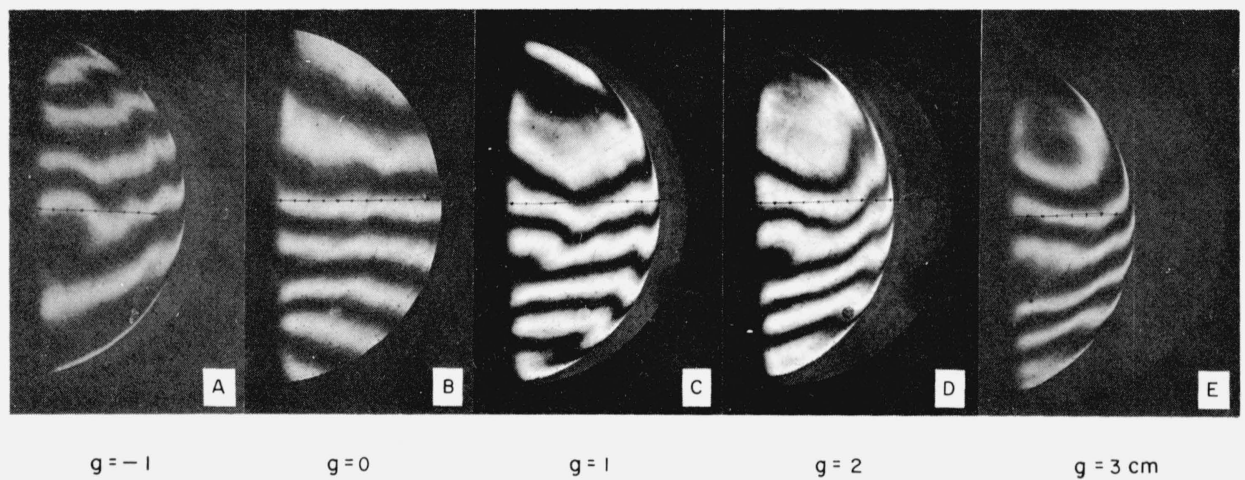

FIGURE 5. Interferograms with Lens $I$, adjusted for $\beta$ (or $\mathrm{h})=0, \theta=0$, and to different values for $\mathrm{g}$.

best value for $A$. To obtain this best value would require least squares analysis. However, since this paper is intended only to show procedures and the good agreement found between this interferometer and other methods of test, a graphical determination of $A$, is assumed to be adequate.

Values for $A$, corresponding to different values for $h$ are plotted in figure 9. The slope of the best fitting straight line is (eqs 5 and 10$)$ equal to $\left(a_{2} / f\right) \cos \theta$. Multiplying the slope by $f / \cos \theta$ gives the value $a_{2}$. The same procedure is used for obtaining $a_{1}$. In this case $A$ is plotted against $g$, (eqs 5 and 9) for constant values of $h$.

Table 3 shows readings from the interferogram, read along the line $Y=5 \mathrm{~cm}$ (at different values of $X$ ) for pairs of points that are equal distances from the $Y$-axis but of opposite sign. The corresponding mean values of $\left(P_{12}-P_{34}\right) / 4 Y$ are shown in column 5 . From eq 8 , the slope, $B$, of the straight line $\left(P_{12}-P_{34}\right) / 4 Y$ $=B X$ (fig. 10) is found to be $-0.126(P / 2) \mathrm{cm}^{2}$. The mean value for $B$, in eq 6 (for $h=h$ and $h=-h$ ), is plotted against $h^{2}$ in figure 11. The slope of the corresponding best line is $\left(a_{3} / f^{2}\right)$ sin $2 \theta$, from which $a_{3}$ is obtainable.

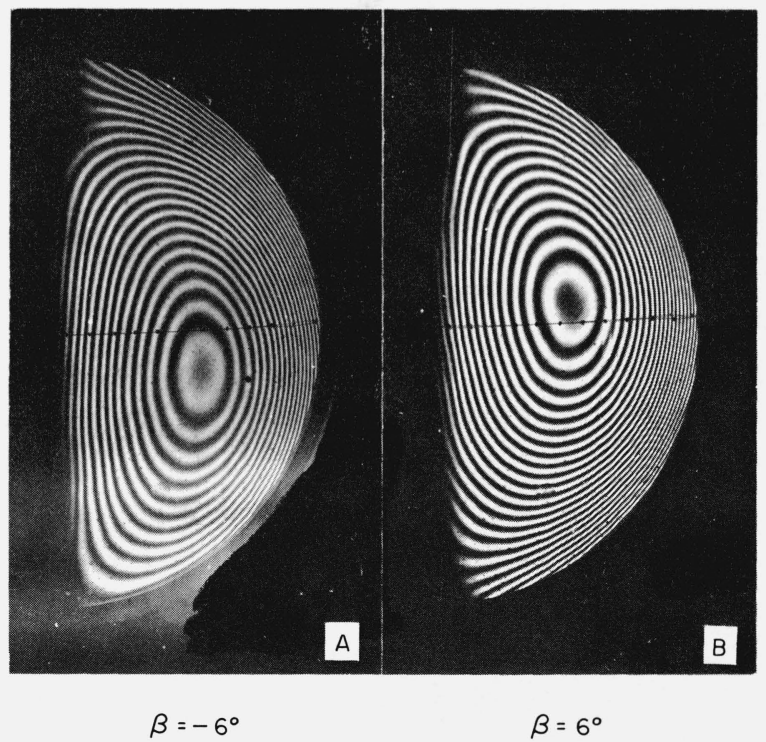

FiguRE 6. Interferograms with Lens No. I, adjusted to $\mathrm{g}=0, \theta=45^{\circ}$, and to different values for $\beta$. 


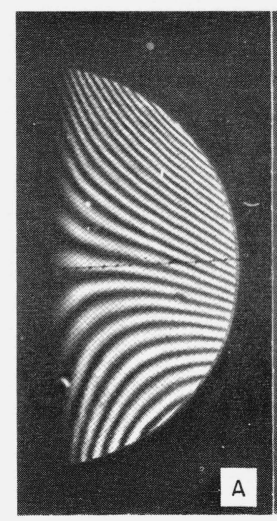

$\beta=-2^{\circ}$
B

$\beta=-1^{\circ}$

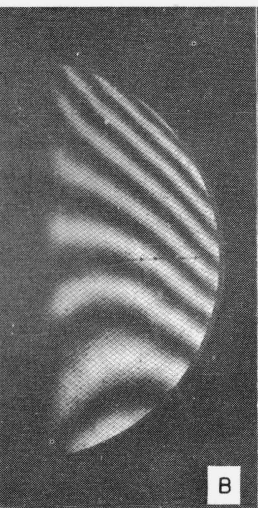

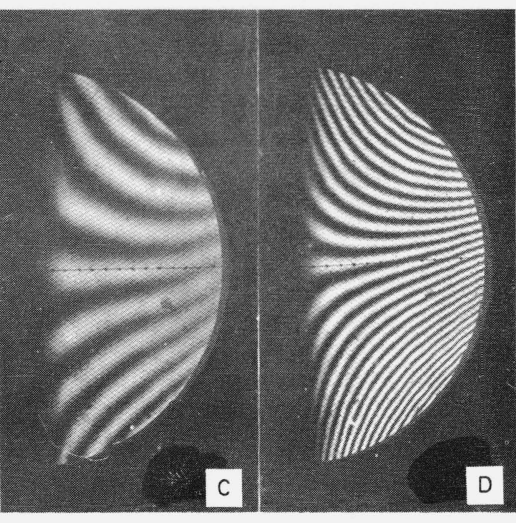

$\beta=1^{\circ}$

$\beta=2^{\circ}$

FiguRe 7. Interferograms with Lens No. $I$, adjusted to $\mathrm{g}=0, \theta=0$ and to different values for $\mathrm{h}($ or $\beta)$.

TABLE 2. Example for computing $A$

Prism and lens adjustments: $g=0, \beta=6^{\circ}$, and $\theta=0$.

\begin{tabular}{c|c|r|r}
\multicolumn{4}{c}{ Reference points on $Y$-axis $(X=0)}$. \\
\hline$Y$ & \multicolumn{1}{c|}{$N$} & \multicolumn{1}{c}{$P$} & $P / 2 Y$ \\
\hline 0 & -0.6 & 0.0 & $\ldots \ldots \ldots$ \\
1 & -3.9 & -3.3 & -1.65 \\
2 & -6.9 & -6.3 & -1.57 \\
3 & -9.2 & -8.6 & -1.43 \\
4 & -11.7 & -11.1 & -1.39 \\
5 & -13.3 & -12.7 & -1.27 \\
6 & -14.1 & -13.5 & -1.12 \\
7 & -14.1 & -13.5 & -0.97 \\
8 & -13.1 & -12.5 & -.78 \\
9 & -11.0 & -10.4 & -.58 \\
10 & -7.0 & -6.4 & -.32 \\
11 & -2.5 & -1.9 & -.09 \\
12 & +3.5 & +4.1 & +.17 \\
\hline
\end{tabular}

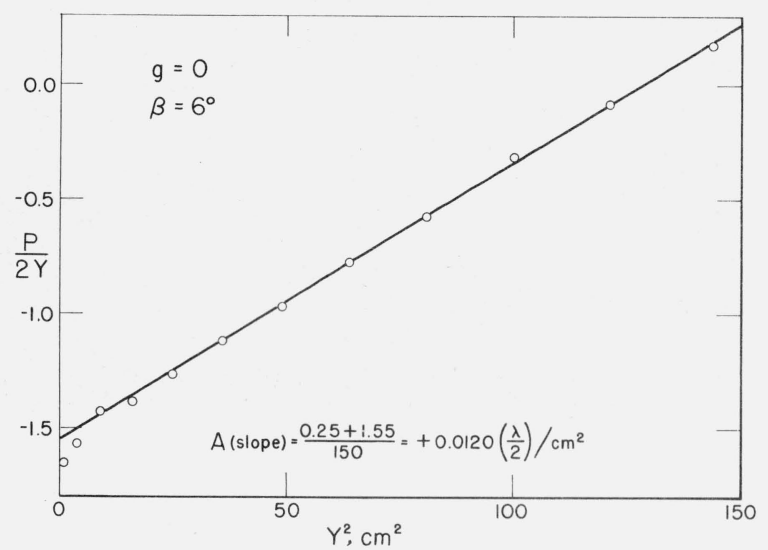

Figure 8. Graphical determination of A from eq (4).

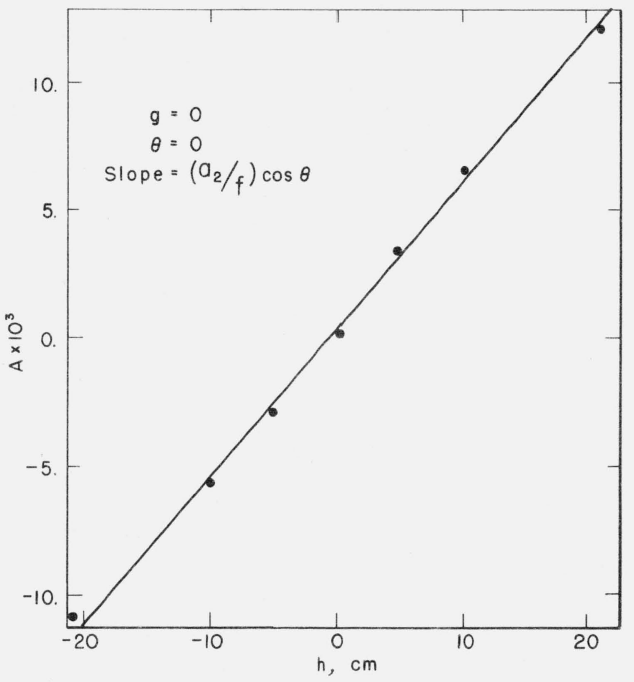

FiguRE 9. Graphical determination of $\mathrm{a}_{2}$ from eqs (4), (5), and (10).

TABLE 3. Data from figure $6 a$ and computed results for figure 8

\begin{tabular}{l|r||c|c|c}
\hline \hline$X$ & \multicolumn{1}{|c||}{$N$} & $X$ & $N$ & $\left(P_{12}-P_{34}\right) / 4 Y \mathrm{~cm}$ \\
\hline $0 \mathrm{~cm}$ & 0.8 & $\mathrm{~cm}$ & & \\
2 & -1.6 & -2 & 3.5 & -0.26 \\
4 & -3.8 & -4 & 6.4 & -.51 \\
6 & -5.5 & -6 & 9.4 & -.76 \\
8 & -7.0 & -8 & 13.0 & -1.00 \\
10 & -8.2 & -10 & 17.0 & -1.26 \\
\hline
\end{tabular}




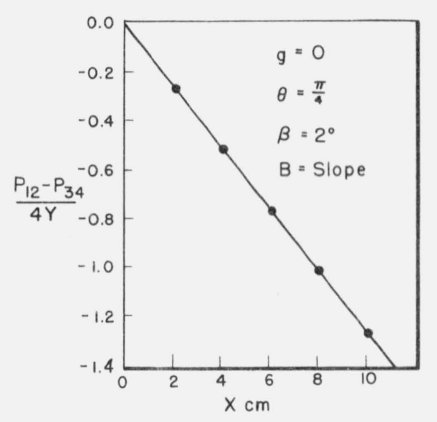

F IGURE 10. Graph for evaluating B from eq (9).

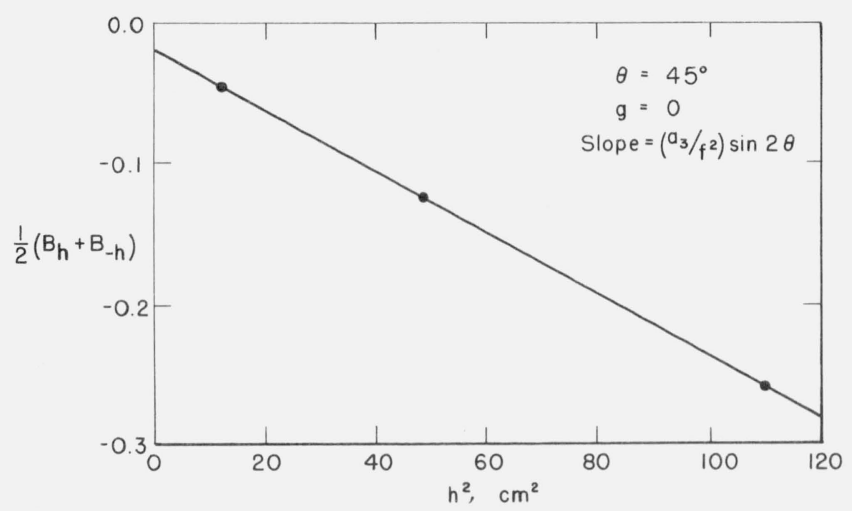

FigURE 11. Graphical determination of $\mathrm{a}_{3}$ from eq (5).

\section{Results}

The data and computation used to make the comparison of results by interferometry with those from other methods is too extensive to be included here, except in graphical and tabular forms. The geometrical method used for obtaining aberration constants will not be described. There are several such methods available. The Foucault knife edge, for example is one of the simplest of methods for measuring spherical aberration. When the lens specifications are known the aberrations can be computed from theory.

\subsection{Lens No. I (Aspherical, Plano-Convex)}

When only spherical aberration exists in an interferogram (i.e., $h=0$ ) and if the lens is corrected for this aberration, the observed value for this aberration will be very small. In this case the higher order aberrations are dominant and eq (7) appears not to satisfy the data. Figure 12 shows a plot of $\mathrm{P} / 2 \mathrm{Y}$ versus $Y^{2}$ (eqs 4 and 7 ) for a constant value of $X$. The slope of this curve (values for $A=a_{1} g$ ) is small and varies uniformly over a small range.

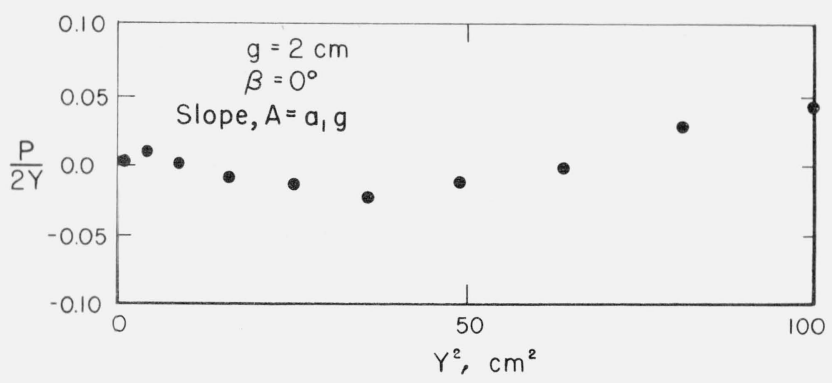

Figure 12. Graphical representation of eq (4), showing nonlinearity and, consequently, nonconformity to the basic optical path difference eq (1).

TABLE 4. Computation of $\mathrm{a}_{2}$ for Lens I

\begin{tabular}{c|r||c|c}
\multicolumn{4}{c}{ Adjustments: $g=0$ and $\theta=0}$. \\
\hline \hline$\beta$ & $\mathrm{A} \mathrm{N} / 2 \mathrm{~cm}$ & $\beta$ & $\mathrm{A} \mathrm{N} / 2 \mathrm{~cm}$ \\
\hline & & & \\
$1.5^{\circ}$ & 0.0032 & $-1.5^{\circ}$ & -0.0029 \\
$3.0^{\circ}$ & .0065 & $-3.0^{\circ}$ & -.0058 \\
$6.0^{\circ}$ & .0122 & $-6.0^{\circ}$ & -.0111 \\
\hline
\end{tabular}

The data for evaluating $a_{2}$ and $a_{3}$, from lens No. I, fall very nearly on a straight line. The resultant values for these constants are very good. Table 4 and figure 9 show the $A$ 's for different values of $h$ that are used to evaluate $a_{2}$. The slope, $\left(a_{2} / f\right) \cos \theta$, of the straight line in figure 9 is found to be $0.000570(\lambda / 2) / \mathrm{cm}^{3}$, from which we obtain:

$$
a_{2}=3.42 \times 10^{-6} / \mathrm{cm}^{2} .
$$

Table 5 and figure 11 show the corresponding data and results for $B$. The slope $B=\left(a_{3} / f^{2}\right) \sin 2 \theta$, of the straight line in figure 11 is found to be -0.00216 $(\lambda / 2) \mathrm{cm}^{4}$, from which we obtain: $a_{3}=2.59 \times 10^{-3} / \mathrm{cm}$.

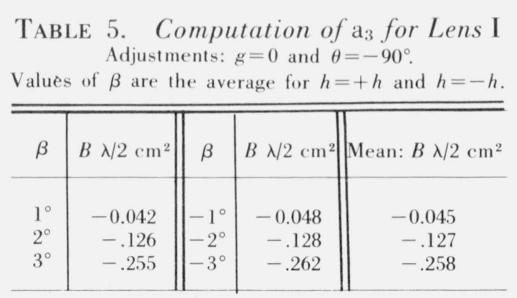

Table 6 is a summary of results obtained with the interferometer, results by direct (geometrical) measurements, and values obtained from theory by computation. The computed values are based on third order aberration theory, assuming the lens to be plano-convex in form. Coma and spherical aberration were too small to be measured directly. These values are considered to represent good agreement between the different methods of test. 
TABLE 6. Summary of results for Lens I

\begin{tabular}{|c|c|c|c|}
\hline Coefficients & $a_{1} \times 10^{7} \mathrm{~cm}^{3}$ & $a_{2} \times 10^{6} \mathrm{~cm}^{2}$ & $a_{3} \times 10^{3} \mathrm{~cm}$ \\
\hline Interferometer method. & Very small & 3.42 & 2.59 \\
\hline Geometrical method......... & & & 2.74 \\
\hline Values computed from theory... & 1.37 & 3.44 & 2.50 \\
\hline
\end{tabular}

TABLE 7. Comparison of results by interferometry, with geometrical measurements, and values computed from theory

\begin{tabular}{c|c|c|c}
\hline \hline \multirow{3}{*}{$\beta$} & \multicolumn{3}{|c}{ Distance between focal lines, Lens I } \\
\cline { 2 - 4 } & Interferometry & Direct & Computed \\
\hline & & & \\
& Millimeter & Millimeter & Millimeter \\
$1^{\circ}$ & 0.63 & $\ldots \ldots \ldots \ldots . .$. & 0.61 \\
$2^{\circ}$ & 2.53 & 2.8 & 2.44 \\
$3^{\circ}$ & 5.69 & 5.7 & 5.49 \\
$4^{\circ}$ & 10.13 & 10.5 & 9.78 \\
\hline
\end{tabular}

The distance between the two astigmatic focal lines was measured directly, for several values of $\beta$. These values are compared with corresponding values computed from interferometer measurements and from theory. Table 7 summarizes these results.

\subsection{Lens No. II, a Telescope Objective}

When values for $P / 2 Y$, in eq (4), are plotted against $Y^{2}(X=$ constant $)$, for obtaining $A$, the points fall on a relatively straight line for values of $Y$ from 0 to $10 \mathrm{~cm}$. Beyond $10 \mathrm{~cm}$, they show deviations that indicate an increasing effect of higher order aberrations. The results for $g=1.5 \mathrm{~cm}$ are shown in figure 13 to illustrate this effect. When the aperture of this lens is limited to $10 \mathrm{~cm}$ the resultant slope of the best fitting straight line yields a value of $1.82 \times 10^{-8} / \mathrm{cm}^{3}$ for $a_{1}$.

The results for coma and astigmatism, for lens II, are presented in figures 14 and 15, respectively. Table 8 summarizes the results for lens II and includes corresponding results obtained by direct measurement. Coma was too small to be measured at the angles used in these tests.

A series of direct measurements was also made on the distance between focal lines (astigmatism) for this lens and the results are compared, in table 9, with results obtained from interferometer tests of the same angles (values for $\beta$ ).

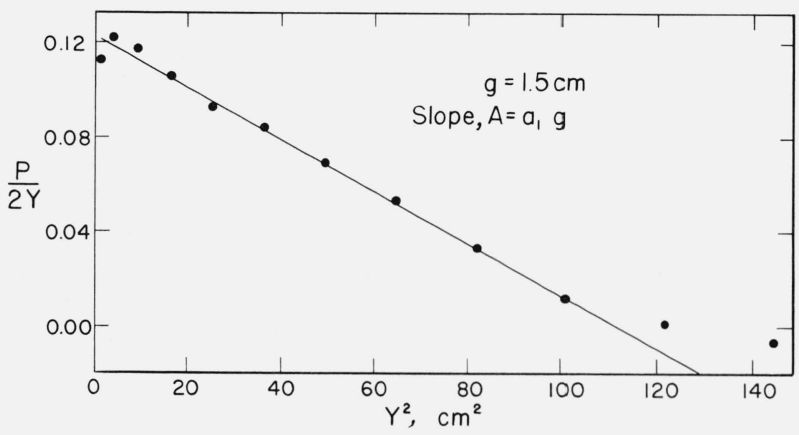

Figure 13. Graph for evaluating $\mathrm{a}_{1}$ of Lens No. II.

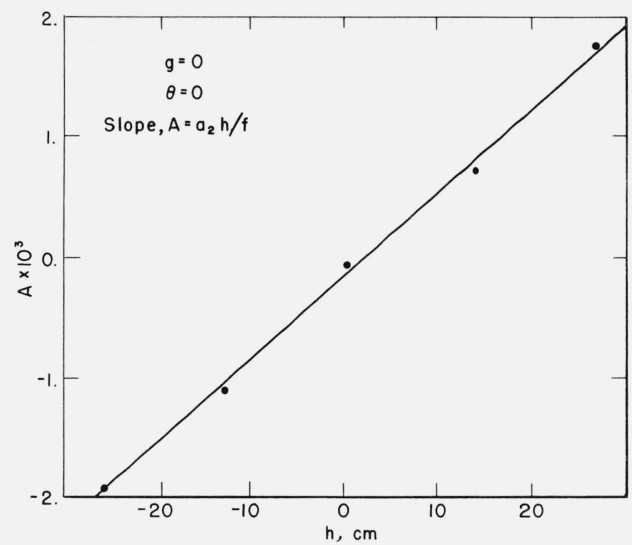

FiguRE 14. Graph for evaluating $\mathrm{a}_{2}$ of Lens No. II.

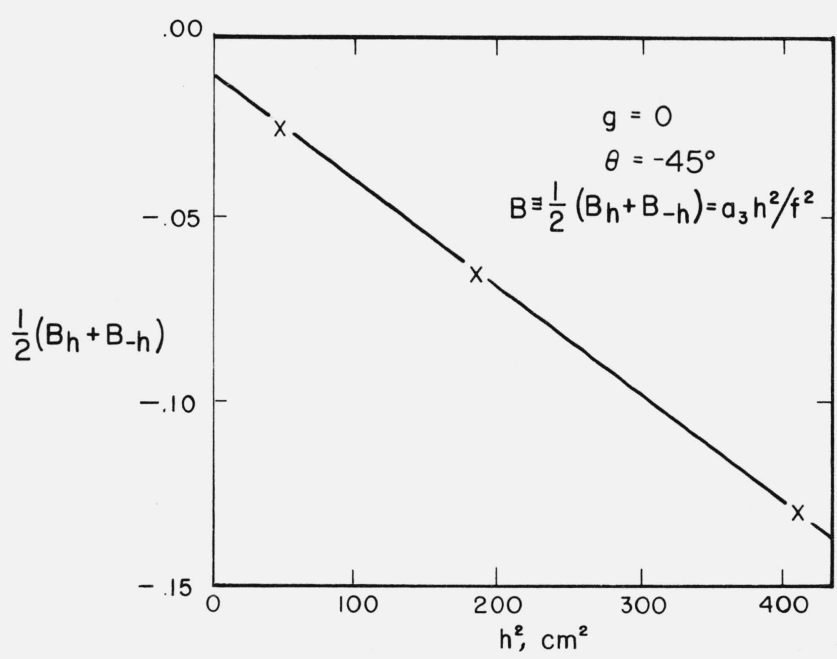

FigURE 15. Graph for evaluating $\mathrm{a}_{3}$ of Lens No. II.

TABLE 8. Summary of results for Lens II

\begin{tabular}{|c|c|c|c|}
\hline Coefficients & $a_{1} \times 10^{8} \mathrm{~cm}^{3}$ & $a_{2} \times 10^{7} \mathrm{~cm}^{3}$ & $a_{3} \times 10^{3} \mathrm{~cm}$ \\
\hline $\begin{array}{l}\text { Interferometer method..... } \\
\text { Geometrical method......... }\end{array}$ & $\begin{array}{l}1.82 \\
1.93\end{array}$ & 7.71 & $\begin{array}{l}1.28 \\
1.32\end{array}$ \\
\hline
\end{tabular}

TABLE 9. Comparison of results by interferometry with results from direct measurements

\begin{tabular}{c|c|c}
\hline \hline \multirow{3}{*}{$\beta$} & \multicolumn{2}{|c}{ Distance between focal lines, Lens II } \\
\cline { 2 - 3 } & & Geometrical \\
\cline { 2 - 3 } & Interferometry & \\
& & $\mathrm{cm}$ \\
$1^{\circ}$ & $\mathrm{cm}$ & 0.11 \\
$2^{\circ}$ & .46 & .50 \\
$3^{\circ}$ & 1.04 & 1.06 \\
$4^{\circ}$ & 1.84 & 1.92 \\
\hline
\end{tabular}




\section{Chromatic Aberration}

The change in focal distance, with change in wavelength of the light, can be measured very accurately with this interferometer. If the dividing plane of the prism is adjusted to pass through the focal point, $f_{1}$, of the lens for light of wavelength $\lambda_{1}$ (see fig. 16), and to form an angle $\alpha$ with the optic axis, $0 f_{1}$, of the lens the light for other wavelengths will not return toward $f_{1}$. If $f_{2}$ is the focal point for light of wavelength $\lambda_{2}$, this light will return toward the point $I_{2}^{\prime}$, where the distance $\left(f_{1}-f_{2}\right)$ equals $\left(f_{2}-I_{2}^{\prime}\right)$. The points $f_{2}$ and $I_{2}^{\prime}$ are on the axis of the lens but not in the dividing plane. The two component beams of $\lambda_{1}$ (one on each side of the dividing plane) will return toward $f_{1}$. They will form two images at $M_{2}$ in figure 2 that are superimposed. The two component beams of $\lambda_{2}$ will form two separated images at $I_{2}^{\prime}$ and $I_{2}^{\prime \prime}$.

Figure 16 illustrates the relative position of points and lines in the image space. The distance $\left(f_{1}-I_{2}^{\prime}\right)$ is approximately equal to $2\left(f_{1}-f_{2}\right)=2 \Delta f$. If the dividing plane intersects the lens at $S$, which is at a distance $g$ from its axis, the angle $\alpha$ is given by the formula,

$$
\alpha=g / f=d /\left(f_{1}-I_{2}^{\prime}\right)=d / 2 \Delta f .
$$

It should be remembered that the angles $\epsilon$ and $\alpha$ (fig. 16) are relatively small and that the distances, $g$ and $L$, are also small compared to the focal length $f$. The distance, $L$, is the distance from the dividing plane at $S$ to an arbitrarily chosen reference point, $T$. If the observed difference in order of interference, between points $S$ and $T$, is $N$ then the angle $\epsilon$ is, given by the formula,

$$
\epsilon=d / f=N \lambda_{2} /(2 L) .
$$

On eliminating $d$ from eqs (12) and (13), we obtain the working formula,

$$
\Delta f=f^{2} N \lambda_{2} /(4 g L)
$$

All quantities on the right hand side of this formula may be measured and, consequently values for $\Delta f$ are obtainable for any chosen wavelength.

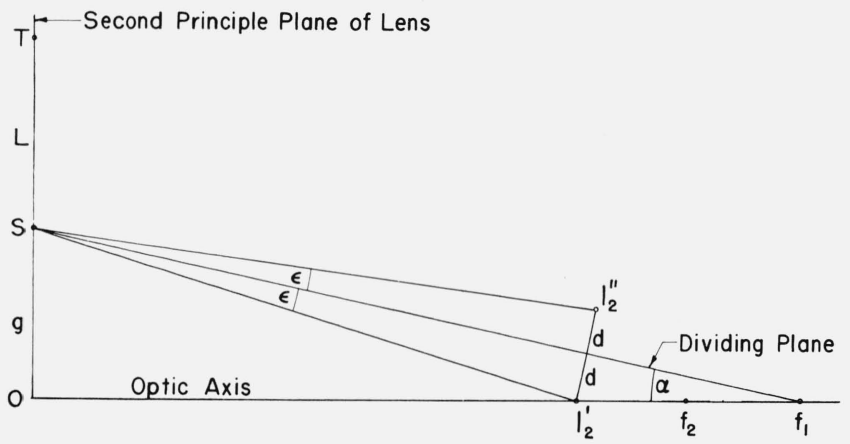

FIGURE 16. Relative position of points and lines in the image space.
For visual observations it is convenient to set the dividing plane at some chosen wavelength and observe values of $N$ for all other wavelengths that are to be used. However, for photographing the fringes it was found more convenient to adjust the dividing plane visually at each of the several focal points and to use the yellow line of helium for photographing the fringes. These photographs permit a measure of the difference in focal length of the lens, for the light chosen, and that for helium yellow $(\lambda=0.5876 \mu)$.

Figure 17 shows several photographs of fringes produced by light of different wavelengths. The number of fringes in these pictures is a measure of the difference in focal length of lens $\mathrm{I}$, for $\lambda=0.5876 \mu$ and for the indicated wavelengths. Figures 18 and 19 are graphical representations of changes in focal length of lens I and lens II, respectively. There is also included for comparison, values obtained by direct measurement of the focal distances.

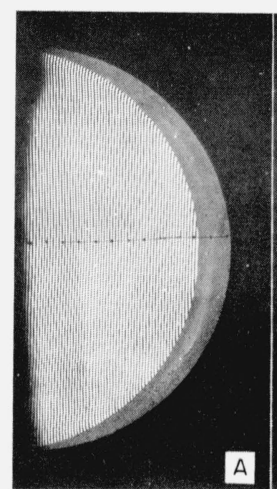

$\lambda=4471$

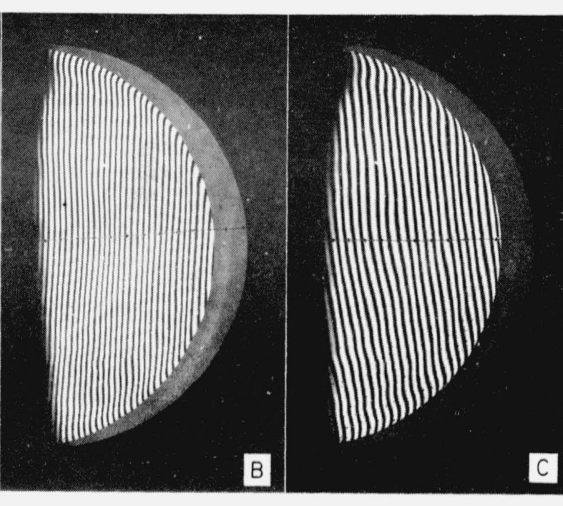

$\lambda=5016$
Figure 17. Photographs of fringes, with yellow light, when the prism is adjusted for autocollimation of light of the several indicated wavelengths.

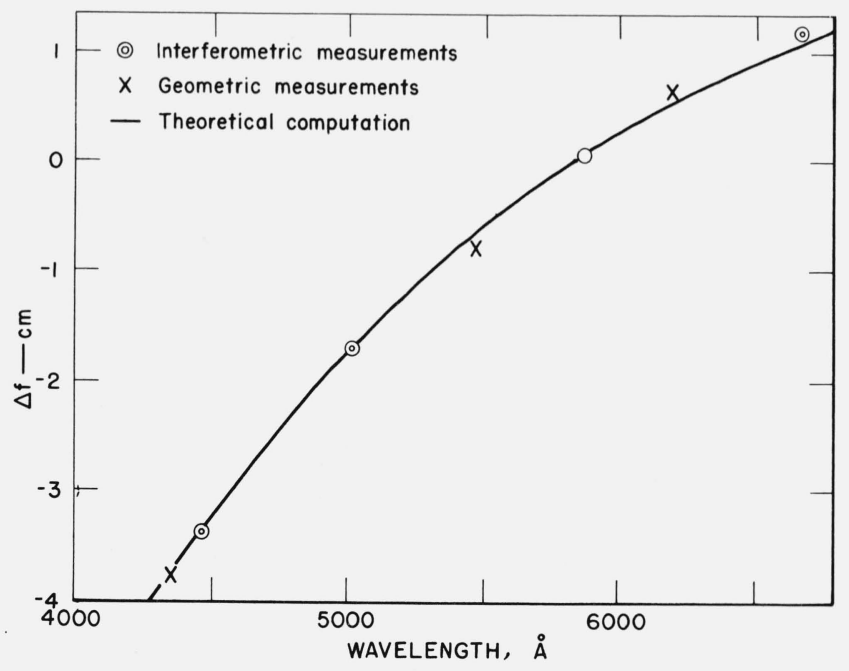

Figure 18. A plot of focal length of Lens I versus wavelength of light. 


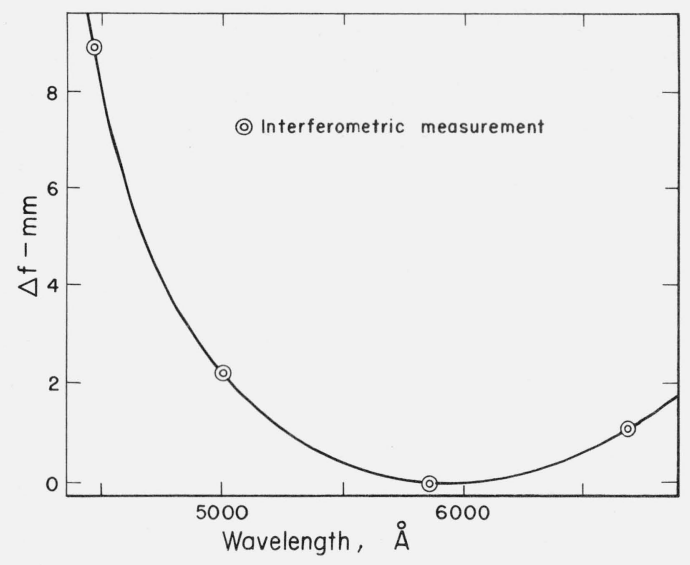

Figure 19. A plot of focal length of Lens II versus wavelength of light.
This study was done under the guidance of J. B. Saunders, Engineering Metrology Section, to whom the author would like to express his sincere thanks for his kind instructions including arranging the present report. He is also indebted to I. C. Gardner for his ceaseless encouragement.

\section{References}

[1] J. B. Saunders, Construction of a Kösters double-image prism, J. Res. NBS 58, 2l(1957) RP2729.

[2] J. B. Saunders, The wavefront reversing interferometer: reprinted from Optics in Metrology (Pergamon Press, London, England, 1960).

[3] J. B. Saunders, Optical Society of America, 44th Annual Meeting, Lake Placid Club, Essex County, New York: Oct 18, 19 \& 20, 1956. Paper No. SA 12.

[4] R. Kingslake, Trans. Opt. Soc. 27, p. 95 (1925-6).

[5] J. B. Saunders, The Kösters interferometer, J. Res. NBS 58 , 27 (1957) RP2730.

(Paper 70C2-220) 